\title{
Primordial black holes and asteroid danger
}

\author{
Alexander Shatskiy * \\ * Astro Space Center of the P. N. Lebedev Physics Institute, Russian Academy of Sciences \\ ul. Profsoyuznaya 84/32, 117997 Moscow, Russian Federation
}

\begin{abstract}
Probability for a primordial black hole to invade the Kuiper belt was calculated. We showed that primordial black holes of certain masses can significantly change asteroids' orbits. These events may result in disasters, local for our solar system and global for the Earth (like the Tunguska meteorite). We also estimated how often such events occur.
\end{abstract}

\section{Introduction}

Nowadays the asteroid danger is one of the most important scientific issues. Geophysical data indicate that in the past the Earth was dangerously attacked by asteroids about every 190 million years rather than continuously. These repeated global events suggest that the probability for asteroids, or meteorites, to shoot is not constant, but depends on external (with respect to the Solar system) factors (see [1]).

At the moment the nature of dark matter is not clear. One of the candidates for dark matter particle is a primordial black hole $(\mathrm{PBH})$. In case the entire dark matter consists of $\mathrm{PBHs}$, it is possible to set not only the lower (Hawking) bound for their masses, but also the upper one. The latter comes from the data on gravitational microlensing (for example, see [2]). However, the upper bound is quite uncertain and in different estimations lies in the range $10^{26} \div 10^{28}$ g, i.e. close to the Earth and Moon masses.

In this paper we obtained the probability for a $\mathrm{PBH}$ travelling through the Solar system to collide (interact) with an asteroid. This process can make collision of the Earth and some heavenly body more probable.

To date, we are aware of several asteroids whose orbits are threateningly close to the Earth. The asteroids that are sufficiently big and massive are most dangerous to our planet. For example, the Tunguska meteorite must have been around $50 \mathrm{~m}$ in diameter.

As is well-known, there are three prominent asteroid belts in the Solar system:

1. Mass of the main asteroid belt (the Phaeton belt) is about $10^{24} \mathrm{~g} \sim 10^{-3} M_{\oplus}$. Asteroids populating the belt are 2.5-3 astronomical units (AU) away from the Sun.

2. Mass of the Kuiper belt is around $10^{27} \mathrm{~g} \sim M_{\oplus}$. Its asteroids are at the distance of 40-60 AU from the Sun.

3. The Oort cloud is located on the Solar system border (more than $100 \mathrm{AU}$ ), and its mass estimations are rather vague.

As is known, probability to collide with a heavenly body is in direct proportion with its surface area. Total mass of the asteroids is comparable with mass of the Earth, but since

*e-mail: shatskiy@asc.rssi.ru 
there are so many of them, their overall surface area appears to be almost equal to the value for the Sun. Besides, there is one more factor which increases the collision probability. An asteroid mass is considerably smaller than that of the Sun. This implies that collision with a PBH should not be necessarily direct to change the asteroid's trajectory. In order to accomplish this, the PBH should only be gravitationally scattered on the asteroid, i.e. it should pass by the asteroid at some distance. This phenomenon increases the probability for a $\mathrm{PBH}$ and an asteroid to interact as much as for several orders of magnitude.

Thus, PBHs interact much more probably with asteroids than with the planets or even the Sun.

\section{Probability to find a $\mathrm{PBH}$ in the Solar system}

According to our hypothesis we suppose that PBHs are responsible for almost entire mass of our Galaxy. Simply put, we set the same mass $m_{p b h}$ for every PBH (in a more complicated case one can just introduce a distribution function of the $\mathrm{PBH}$ mass $m_{p b h}$ ).

The PBH concentration in the Galaxy is $n_{p b h}=\rho_{\text {gal }} / m_{p b h}$, where $\rho_{g a l} \approx 10^{-24} \mathrm{~g} / \mathrm{cm}^{3}$ is the average mass density in the Galaxy.

Therefore, the frequency of the Solar system - $\mathrm{PBH}$ collisions is

$$
\nu_{0}=\pi R_{a}^{2} v \rho_{g a l} / m_{p b h},
$$

where $R_{a}$ is the average radius of an asteroid orbit, and $v$ is the average asteroid velocity in the Galaxy (at infinity with respect to the Solar system).

\section{Probability for a PBH to collide with an aster- oid}

Let the total mass of the asteroids be $M_{\text {ast }}$. Density of an asteroid is usually in the following range: $\rho_{\text {ast }}=2 \div 7 \mathrm{~g} / \mathrm{cm}^{3}$. Asteroid sizes cover a large interval, with most of the rocks having less than $1 \mathrm{~km}$ in diameter and smaller size asteroids being more abundant. Since small asteroids do not result in significant impact, they are not interesting to deal with. So it makes sense to consider asteroids bigger than some critical size, e.g. $D_{\text {ast }} \sim 100 \mathrm{~m}$. One can estimate their number as

$$
N_{a s t}=\frac{\gamma_{a s t} \cdot M_{a s t}}{\rho_{a s t} \cdot D_{a s t}^{3}}
$$

where $\gamma_{a s t}$ is a mass fraction of the asteroids bigger than $D_{a s t}$ in diameter.

The frequency $\nu$ of the $\mathrm{PBH}$ - asteroid interaction is obtained by multiplying the frequency $\nu_{0}$ by the probability $\nu_{1}$ for the PBH to interact with a Solar system asteroid, which is

$$
\nu_{1}=S_{a s t} /\left(\pi R_{a}^{2}\right), \quad S_{a s t}=N_{a s t} \cdot \pi h^{2},
$$

where $h$ is the maximum impact parameter when the $\mathrm{PBH}$ changes significantly the asteroid trajectory.

Hence, using eq. (1) we obtain:

$$
\nu=\frac{\pi \gamma_{a s t} \rho_{g a l} M_{a s t} v h^{2}}{m_{p b h} \rho_{a s t} D_{a s t}^{3}} .
$$

The quantity $h$ is to be calculated in the next section. 


\section{Abrupt change of asteroid orbits due to scat- tering on PBHs}

With the $\mathrm{PBH}$ - asteroid interaction being the classical two-body problem, it is more convenient to solve it in the center of mass frame.

In this reference frame, it is convenient to introduce the following notation 1 :

$m=m_{a s t} m_{p b h} /\left(m_{a s t}+m_{p b h}\right)$ is the reduced mass,

$P=m v$ is the reduced mass momentum in the center of mass frame,

$L=m v h$ is the angular momentum of the $\mathrm{PBH}-$ asteroid system,

$r_{0}$ is the minimal distance between the $\mathrm{PBH}$ and the asteroid,

$U_{0}=G m_{p b h} m_{a s t} / r_{0}$ is an absolute value of the gravitational interaction energy in the orbit's perihelion,

$E=\frac{1}{2} m v^{2}$ is the total energy of the system,

$A=U_{0} /(2 E)=G\left(m_{p b h}+m_{a s t}\right) /\left(r_{0} v^{2}\right)$ is a dimensionless scattering parameter,

$B=r_{0} / h$ is the ratio of the minimal distance to the impact parameter.

Subtracting the zeroth order $\pi$ and taking into account relations between the orbit parameters, we obtain the angle of the momentum deflection after the $\mathrm{PBH}$ has been scattered by the asteroid [3]:

$$
\Delta \varphi=2 \arccos [1]-2 \arccos \left[\frac{-A}{1+A}\right]-\pi .
$$

As for B, the calculation yields:

$$
B^{2}=\frac{1}{1+2 A}
$$

In the center of mass frame, the change of the momentum is 2 :

$$
(\Delta P)^{2}=2 P^{2} \cdot[1-\cos (\Delta \varphi)] .
$$

Using the simple trigonometry and eqs. (5) and (7), we obtain:

$$
\Delta P=P \cdot \frac{2 A}{1+A} \approx 2 P A=\frac{2 G m\left(m_{p b h}+m_{a s t}\right)}{r_{0} v} .
$$

In the Galaxy the $\mathrm{PBH}$ average velocity is comparable to the star velocity dispersion: $v \approx 300 \mathrm{~km} / \mathrm{s}$. Therefore, the total energy $E$ of the system is much more than the gravitational interaction energy: $E>>U_{0}$, hence, $A<<1, B \approx 1$.

The condition of the crucial alteration of the asteroid orbit is equality between the initial asteroid momentum, $P_{a s t}=m_{a s t} v_{a s t}$, and the gained momentum $\Delta P$ within an order of magnitude:

$$
\alpha P_{\text {ast }}=\alpha m_{\text {ast }} v_{\text {ast }}=\Delta P=\frac{2 P A}{1+A},
$$

where, according to eq. (7), the parameter $\alpha=\sqrt{2(1-\cos \Delta \varphi)}$ reflects how strongly the asteroid is affected.

Taking into consideration that the asteroid velocity on a circular orbit of radius $R$ is $v_{\text {ast }}=\sqrt{G M_{\odot} / R}$ and making use of eq. (9), we obtain the exact expression for $h^{2}$, the mass of asteroid influence having been taken care of:

$$
h^{2}=\frac{4 G m_{p b h}^{2} R}{\alpha^{2} v^{2} M_{\odot}} \cdot\left[1-\left(\frac{\alpha\left(m_{p b h}+m_{a s t}\right) v_{a s t}}{2 m_{p b h} v}\right)^{2}\right],
$$

\footnotetext{
${ }^{1}$ Here, $G$ is the gravitation constant, $M_{\odot}$ is mass of the Sun, $M_{\oplus}$ is mass of the Earth.

${ }^{2}$ An absolute value of the momentum change is the same both for the asteroid and the $\mathrm{PBH}$.
} 
or

$$
h \approx\left(\frac{m_{p b h}}{\alpha M_{\oplus}}\right) \cdot \sqrt{\frac{R}{R_{a s t}}} \cdot 800 \mathrm{~km},
$$

where $R_{a s t} \approx 10^{15} \mathrm{~cm}$ is the average distance to the Kuiper belt.

Using this last but one expression, we obtain for $A$ :

$$
A \approx \alpha \sqrt{\frac{G M_{\odot}}{4 v^{2} R}} \cdot\left(\frac{m_{p b h}+m_{a s t}}{m_{p b h}}\right)<<1 \quad\left(\text { at } m_{p b h}>m_{a s t}\right) .
$$

This last expression validates the above-made assumption on A's being small for most asteroids of the Kuiper belt.

Eqs. (3) and (10) yield the probability for an asteroid to be deflected by a PBH provided the latter has gotten into the Solar system:

$$
\nu_{1} \approx \frac{4 G m_{p b h}^{2} \gamma_{a s t} M_{a s t}}{\alpha^{2} v^{2} M_{\odot} R_{a s t} \rho_{a s t} D_{a s t}^{3}} \approx \frac{2 \cdot \gamma_{a s t} \cdot m_{p b h}^{2} \cdot(100 \mathrm{~m})^{3}}{\alpha^{2} \cdot M_{\oplus}^{2} \cdot D_{\text {ast }}^{3}}
$$

Substituting eq. (10) to eq. (4), we obtain the frequency of significant orbit deviations for big asteroids:

$$
\nu \approx \frac{4 \pi G \gamma_{a s t} \rho_{g a l} M_{a s t} R m_{p b h}}{\alpha^{2} M_{\odot} \rho_{a s t} D_{a s t}^{3} v}
$$

\section{Conclusion}

It is convenient to rewrite the final expression for the frequency of the events under consideration as follows:

$$
\nu \approx\left(\frac{10^{-7} y s^{-1}}{\alpha^{2}}\right) \cdot\left(\frac{m_{p b h}}{M_{\oplus}}\right) \cdot\left(\frac{M_{a s t}}{M_{\oplus}}\right) \cdot\left(\frac{\gamma_{a s t}^{1 / 3} 100 \mathrm{~m}}{D_{a s t}}\right)^{3} \cdot\left(\frac{R}{R_{a s t}}\right),
$$

The last three factors in formula (15) are of the order of unity, so that the final expression only depends on the distribution and total mass of PBHs in the Galaxy.

Estimating the fraction of asteroids hitting the Earth itself is quite difficult. This is due to the fact that, even if the asteroid does not hit some planet, very probably it will not leave the Solar system. Its orbit now crosses orbits of planets and other asteroids and becomes more dangerous. So sooner or later the asteroid itself or secondary asteroids (produced by the very first one) fall onto the planets.

Thus, the probability for the scattered asteroid to hit the Earth can be roughly estimated as the ratio of the Earth surface area to the total surface area of the other planets. It yields approximately $\sim 10^{-2}$. Hence, it does not seem improbable that formula (15) may give hit periods of about 190 million years, which is consistent with observational data.

If the hypothesis analyzed in this paper is correct, modern methods aimed at averting the asteroid danger appear to be inefficient. This is related to the fact that their main idea is revealing big meteors and asteroids with dangerous orbits and, then, monitoring these bodies. However, if the main danger consists in abrupt changes of asteroidal orbits (because of scattering on a $\mathrm{PBH}$ ), revealing potentially dangerous bodies is hardly possible. 


\section{Acknowledgements}

The online materials from the site http://www.ASTROLAB.ru have been used by the author while working on this paper.

\section{References}

[1] Goncharov, G., and Orlov, V., Astron. Rep. 47, No11, p.925, (2003)

[2] Carr, B.J., E-print arXiv: astro-ph/0511743v1, (2005)

[3] Landau, L.D., and Lifshitz, E.M., "Mechanics", Pergamon, Oxford, (1975) 